\title{
Recognising Cortical Sulci and Gyri in MR Images.
}

\author{
C I Attwood, G D Sullivan and K D Baker.
}

\author{
Intelligent Systems Group, \\ Dept. of Computer Science, \\ The University of Reading, RG6 2AY, U.K. \\ Charlie.Attwood@reading.ac.uk
}

\begin{abstract}
We describe an automatic technique for segmenting the outer cortical surface from axial MR images. The scalp outline defines a search area within which the cortex can be expected to lie. The image is sampled radially and a special purpose edge-detector is used to create an initial estimate of the cortical surface. This estimate is then relaxed under the control of local edge constraints. The cortex outline then guides a search for sulci. A physical description of the sulci and gyri of the cortex is derived, which allows a 3D reconstruction of the cortical surface and its sulci to be displayed.
\end{abstract}

\section{Introduction}

The speed and resolution with which MR images of the head can be obtained has increased dramatically recently. Improved availability has led to their increasing use, both as a diagnostic tool and as a means of locating pathologies and hence guiding surgery.

Knowledge of the precise location of cortical sulci and gyri - brain mapping - serves to locate regions of known physiological function. There is much interest, in the pre-operative localisation of "landmark" features such as the lateral sulcus and central sulcus (the two main infoldings of the brain, dividing the frontal, parietal and temporal lobes). Current techniques typically involve obtaining a sequence of slices (the plane depending on the dominant orientation of the sulcus of interest) and then tracing a landmark sulcus manually $[2,4,7,9,10]$.

This paper describes an automatic technique for segmenting the outer cortical surface from axial MR images, which is more robust than the earlier method described in Attwood et al [1]. The cortical surface located serves to guide a search for sulci lying near the brain's surface. The physical descriptions recovered allows a 3D reconstruction of the cortical surface and its sulci to be obtained. The objective is an anatomical labelling of the major sulci, performed automatically. There are however many difficulties due to the wide interindividual variation in sulcus size and relative topology [8].

\section{Cortex Recognition}

The cortex recovery algorithm has been applied to two axial T1 weighted MR data sets. Set 1 , comprising 43 adjacent $2 \mathrm{~mm}$ thick slices, and Set 2 comprising five $8 \mathrm{~mm}$ thick slices, at $4 \mathrm{~mm}$ separation. The cortical boundary is obscured by a number of effects including, partial 


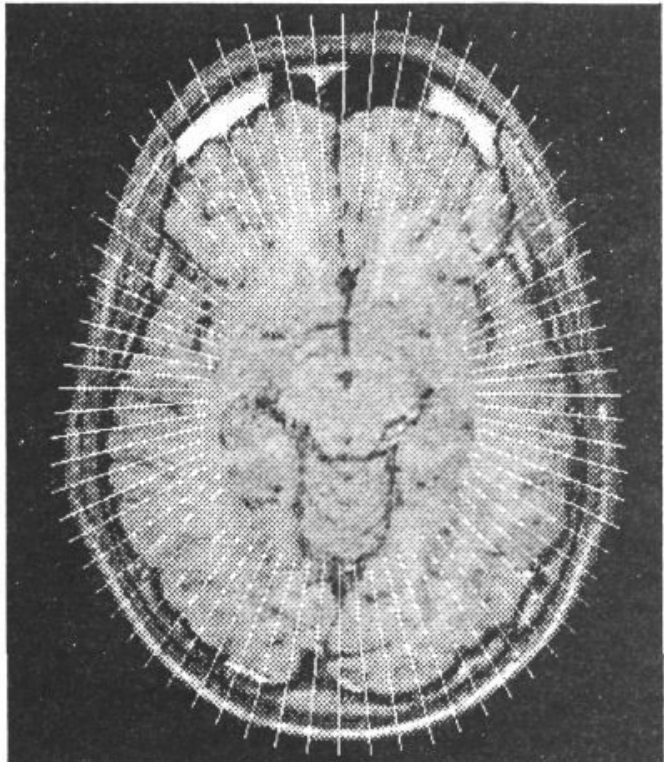

Figure 1. Example radial lines (shown every $5^{\circ}$ ) along which grey values are sampled and cortex search takes place. volume $^{\dagger}$, imager noise and the variety of tissue types which may effectively abut it (cerebro-spinal fluid, blood vessel, bone) creating a variety of edge types.

The technique described here attempts to exploit general properties of brain anatomy, such as its internal homogeneity, approximate circularity and the smooth continuity of the cortical surface.

As a starting point the scalp outline is obtained using an active contour technique [11]. From this, the head centre is calculated for each slice and its average position becomes the central axis of a head-centred coordinate system. Grey values in an image are then sampled (by bi-linear interpolation) along radial lines $(\mathrm{r})$ running from 0.5 to 1.05 of the distance between the central axis and the scalp outline at 360 one

degree intervals $(\theta)$ (see Figure 1). The problem of brain outline recognition is to find the path (in $\mathrm{r}-\theta$ space) which marks the boundary between cortex and other tissues.

The points sampled along each radial are evaluated using a statistical edge detector (SED). A distinguishing feature of the cortex boundary is that it marks a transition between a fairly homogeneous grey area and some new (lighter or darker) grey value. The SED is designed to be sensitive to this structure. Starting from the centre and moving outwards, we compute for each $(\mathrm{r}, \theta)$, the maximum ratio between the running average of the grey values along a radial and the average of the two values in front. This ratio is then signed according to the direction of the grey level slope. This gives a measure of "edgeness" at each point.

\subsection{Seeding The Cortex Search}

Since the distance from the brain centre to the cortex may vary considerably around its perimeter and there are often dark sulci lying interior to the cortex surface which mimic it, a cautious initial estimate of its position is required. $\theta$-space is first divided into four 90 degree sections. For each section a number of simple statistics are calculated including the maximum and the average position of the first edge along each radial which exceeds a fixed threshold. On a second pass, a point of suitable SED strength along each radial, which lies above the mean distance and below the maximum for its section, is accepted as a seed point. Next, any radial for which no seed edge is yet assigned is seeded by selecting a point which minimises the difference in radial position between it and its nearest seeded neighbour, and maximises the SED response. Figure 3 shows an example of the initial SED edges selected.

\footnotetext{
$\dagger$ Partial volume refers to the fact that the grey value of a single pixel is the average over a block of tissue through the thickness of the slice (possibly containing more than one tissue type).
} 
In the majority of instances the cortical boundary is a Grey $\rightarrow$ Dark (-ve) edge. Having obtained seed points (which may include +ve edges), we can refine the -ves further. For each radial we compute the smooth derivative $\frac{d}{d r}\left(G^{*} I\right)$. The radial is then parsed into sequences of $+v e,-v e$ and flat gradient edge points. Negative gradient seed points whose radial position intersects a -ve sequence are shifted to the maximum strength -ve gradient point in that sequence.

\subsection{Cortex Location By Rule-based Relaxation}

After many partially successful attempts at cortex recognition, it became apparent that the algorithm had to incorporate the property of global continuity possessed by the cortical surface, whilst also being sensitive to local discontinuities. We have adopted an iterative relaxation approach. In general terms, local continuity is enforced by distal edge assignments propagating their positional information to those more medial neighbours with a significant difference in radial position. The algorithm attempts to reach a minimum surface tension over the whole cortical surface - thus tending to form a smooth cortex description. The essential steps of the cortex recognition algorithm are summarised in Figure 5 .

Relaxation is performed in $\mathrm{r}-\theta$ space. From an arbitrary start point along the $\theta$ dimension the difference in radial distance (tension) of a seed point and a neighbour is found. If a significant tension exists an attempt is made to reduce it by seeking a new edge assignment. Since the seeding process was deliberately cautious, edge re-assignments are

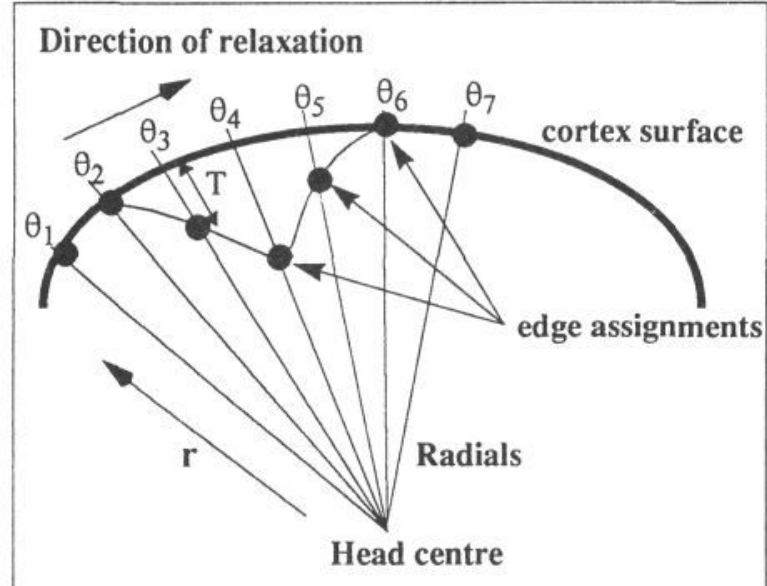

Figure 2. The edge assignment along $\theta_{3}$ is in tension $(T)$ relative to $\theta_{2}$. Tension reduction will attempt to select a new edge further out along $\theta_{3}$ which obeys the necessary constraints. only made outwards, along a radial (see Figure 2).

Prior to tension reduction being attempted, the more distal edge is classified as belonging to one of two categories 1) Grey $\rightarrow$ Dark or 2) Grey $\rightarrow$ Light, based on an examination of the average grey values around it. The area in front of the more medial edge is also evaluated and compared for consistency with its distal partner, as each new edge assignment is evaluated. This information, in conjunction with the degree of tension reduction produced by a new edge assignment, is used to select the best edge re-assignment.

After any re-assignment, the process is repeated at the next $\theta$. This process continues whilst tension reduction is possible, with the direction of travel reversing whenever the distal-medial relationship changes between the current pair of edge assignments. In this way the algorithm sweeps back and forth over areas of surface tension and continues in one direction where the surface is smooth. 


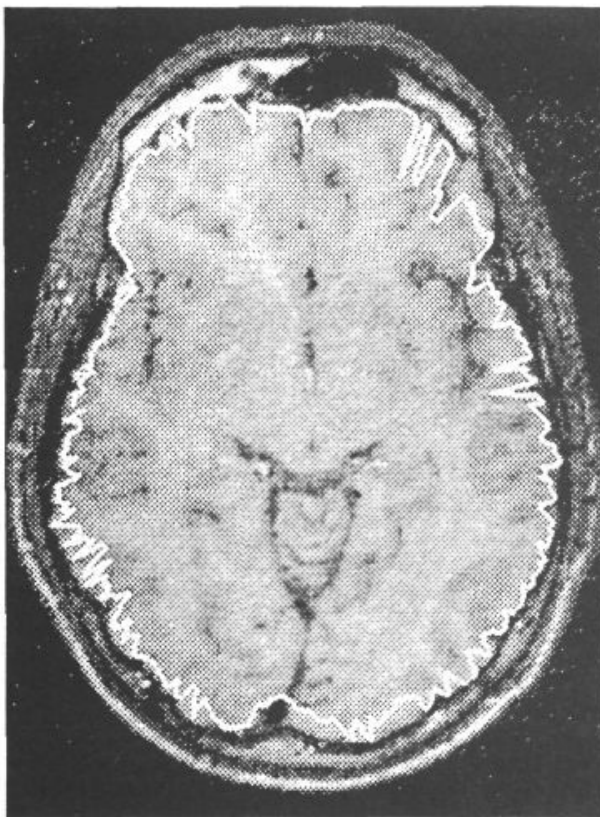

Figure 3. Example of an initial seed from SED edges.

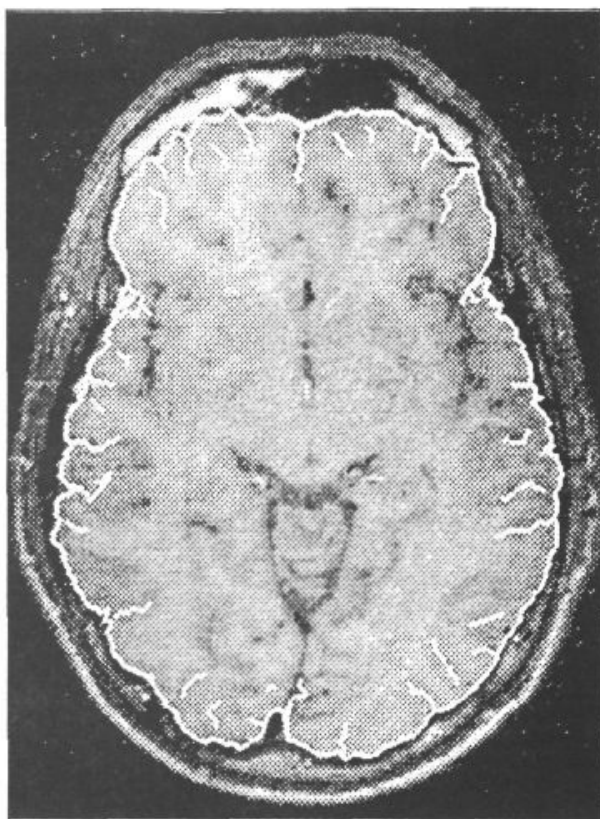

Figure 4. The cortex outline and sulci axes extracted from a typical MR slice.

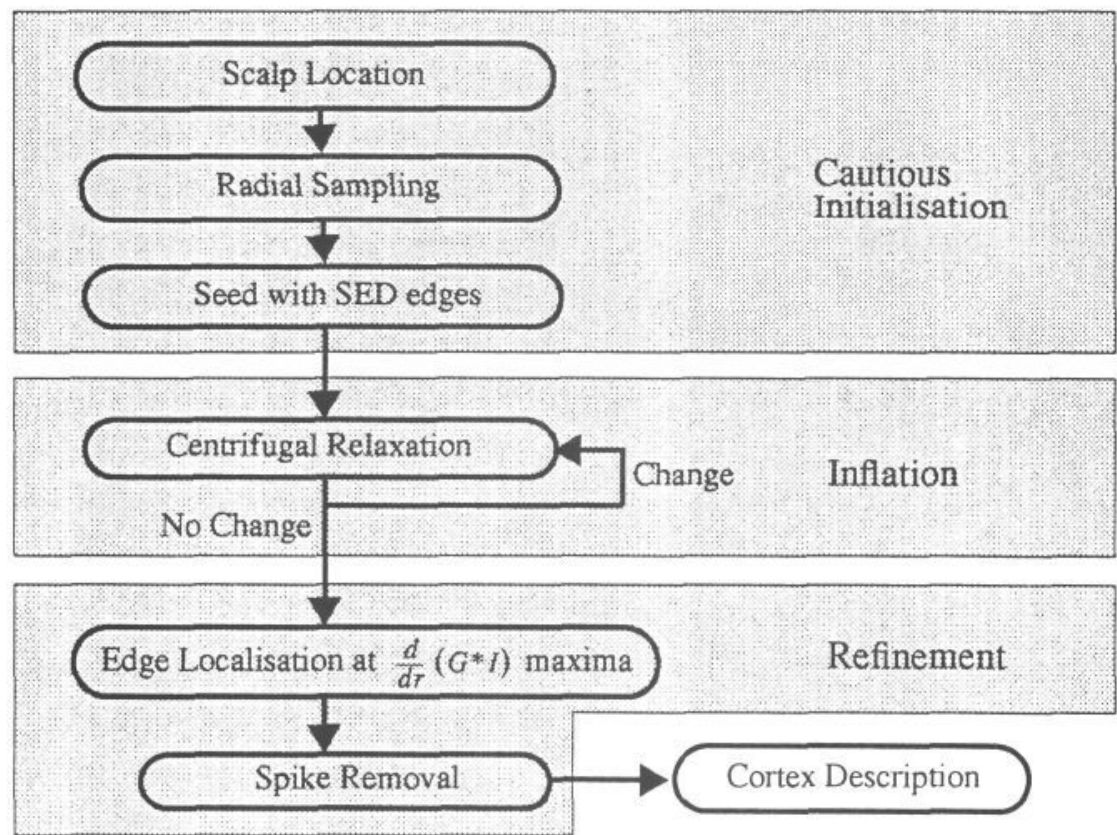

Figure 5. Flow diagram of the basic steps in the cortex recognition algorithm

On subsequent passes in $\theta$-space the new edge assignment may be revised. The relaxation process terminates when a complete 360 degree sweep of the radials is made without any tension reduction occurring. 


\subsection{Refining The Cortex Description}

The SED is effective in capturing the approximate location of significant change points, but lacks the precision which a more local edge detector can provide. Points on the cortex description are revised to the nearest extremum of the smoothed derivative, as in the seeding process. Such revision is only performed if another radial within $\pm 5^{\circ}$ also indicates reassignment. This corroborative check increases confidence that the new assignment is not spurious and helps prevent single unwanted spikes forming. The new edge assignments may have introduced some small new tensions in the cortex surface, so the relaxation process is now repeated.

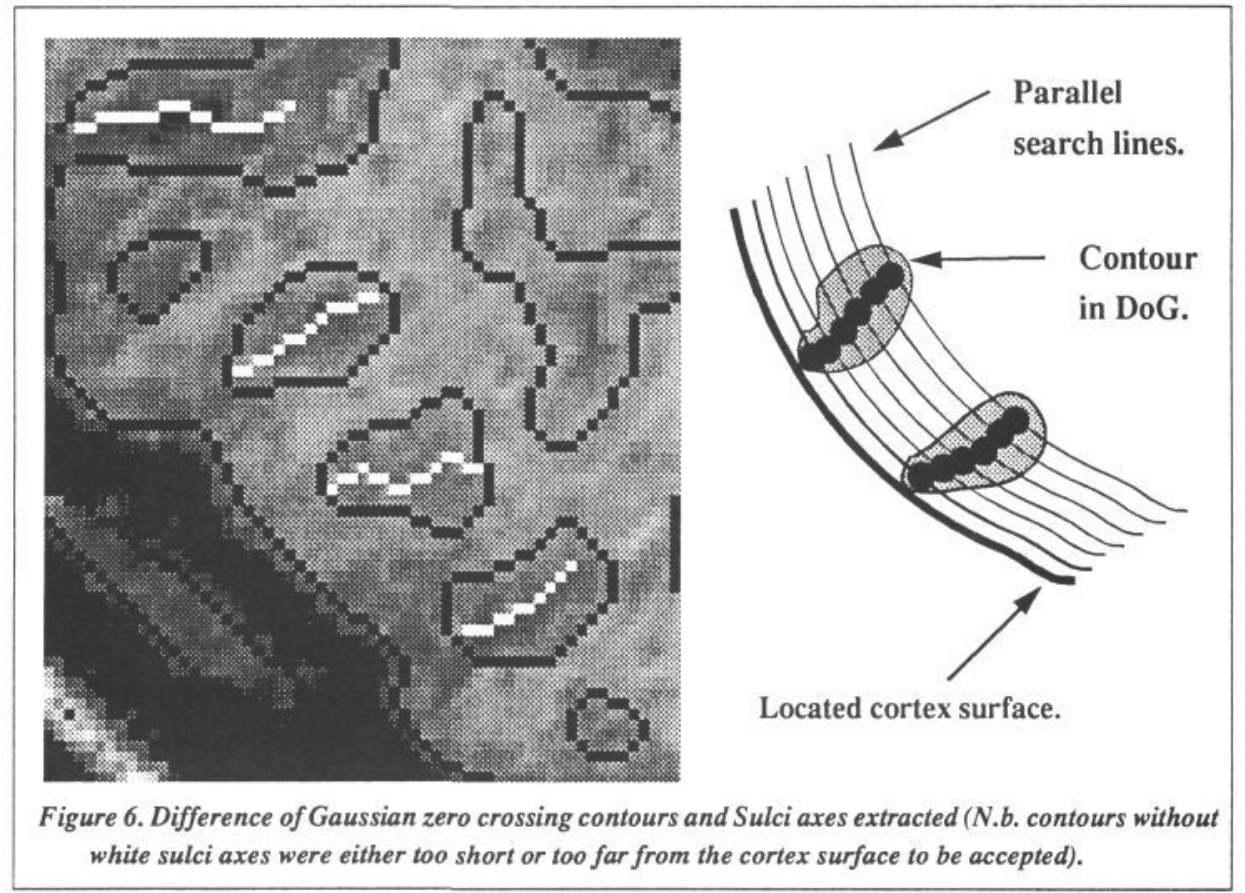

The final step is to look for any residual outward going points of tension in the surface (spikes), unresolved by the centrifugal relaxation process. These are identified as points at which the average grey level immediately interior to the spike is darker than the average grey level immediately exterior to its neighbours. A search is made behind the current edge front for a new SED edge which will reduce the tension and minimise the difference in grey levels. Typical results of the cortex identification process can be seen in Figure 4.

\section{Sulci Location In Axial Slices.}

The cortex identification algorithm produces a kind of "shrink-wrap" description of the outer cortex surface. It worked reliably on 48 images tested from two data sets. Having located the cortex, this relatively coarse scale information is used to guide a search for the sulci lying interior to the cortex surface.

The earlier attempt at sulcus location in Attwood et al [1], parsed a Difference of Gaussian (DoG) zero crossing contour which had been identified as forming a combined description of both the gyri and sulci comprising the cortical surface. Its major limitation was 


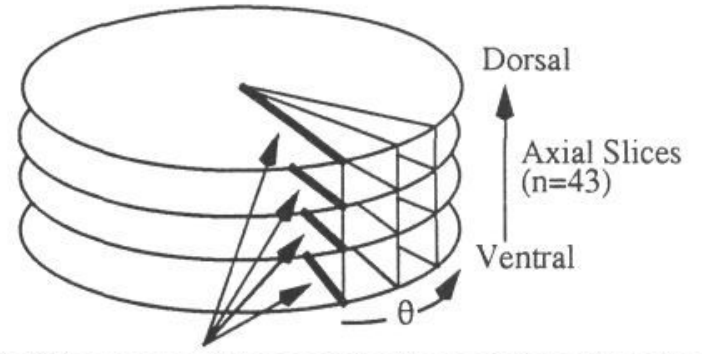

Radials at same $\theta$ in separate slices are joined together to

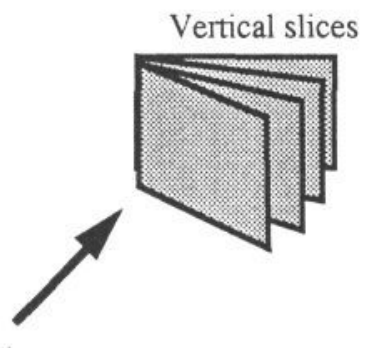
create a series of vertical slices centred on the head's central axis.

Figure 7. Creating vertical slices from radial sample lines.

that small sulci in the original image may give rise to individual "islands" in the zerocrossing map, which are not connected to the main zero crossing contour and may therefore be missed. However the DoG is still potentially useful as a means of recognising indistinct structures such as sulci. The current method makes use of the knowledge gained in locating the outer surface of the cortex, in order to guide the search in the zero crossing map more accurately.

A DoG image, of an appropriate scale $\left(\sigma_{1}=4.0, \sigma_{2}=5.6\right.$ pixels), is first generated. A series of search lines are constructed, lying inside of and parallel to the recovered cortex surface (see Figure 6.). Each search line is traversed and whenever a negative area is entered, a count is started which terminates when the region is left. The mid-point of the dark region is stored, and used to represent a point on a sulcus axis. A search is then made to connect up stored axis points across the search lines using criteria of proximity and minimal angular variation. Those connected sequences coming close to the cortex surface, are accepted as the central axes of sulci.

The result of sulci location for a typical slice is given in Figure 4. Sulci are stored as the polyline description of their axes (in image coordinates) and as the $r-\theta$ value of the mouth of the sulcus.

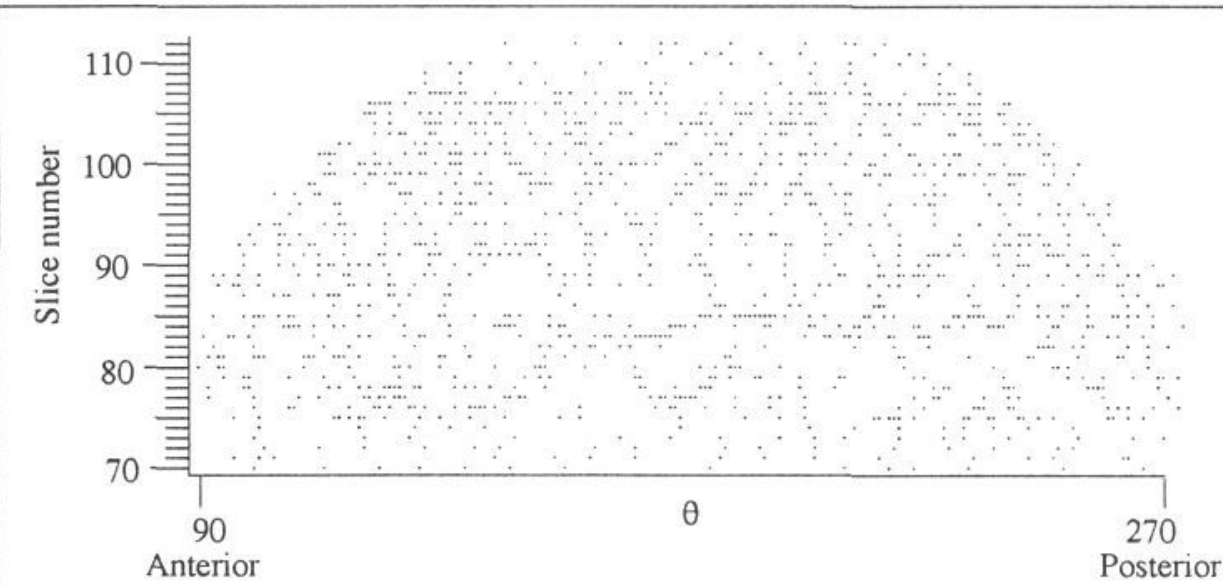

Figure 8. Sulci positions on the surface of the left hemisphere with brain size variation compensated for. 


\section{Sulci Location In Orthogonal Pseudo Slices.}

The algorithm for sulcus location using axial slice data is most sensitive to vertically orientated sulci such as the inferior extent of the pre-central, post-central and central sulci. This is mainly because the partial volume effect is minimised for vertically orientated sulci. Also a sulcus running parallel to the slice plane (for example the lateral sulcus) may appear at different depths inside the cortex according to its angle of inclination, which may lead to the algorithm failing to recognise it. Brain mapping research indicates that vertically oriented slices are best for locating the lateral sulcus $[8,10]$.

By searching for sulci in both horizontally and vertically orientated slices, the location of sulci at all orientations should be improved. Vertical pseudo slices were constructed by resampling, in each axial slice, the grey values found along each radial, and joining the set of cross-slice samples obtained for each theta, into a new image (see Figure 7). By creating a

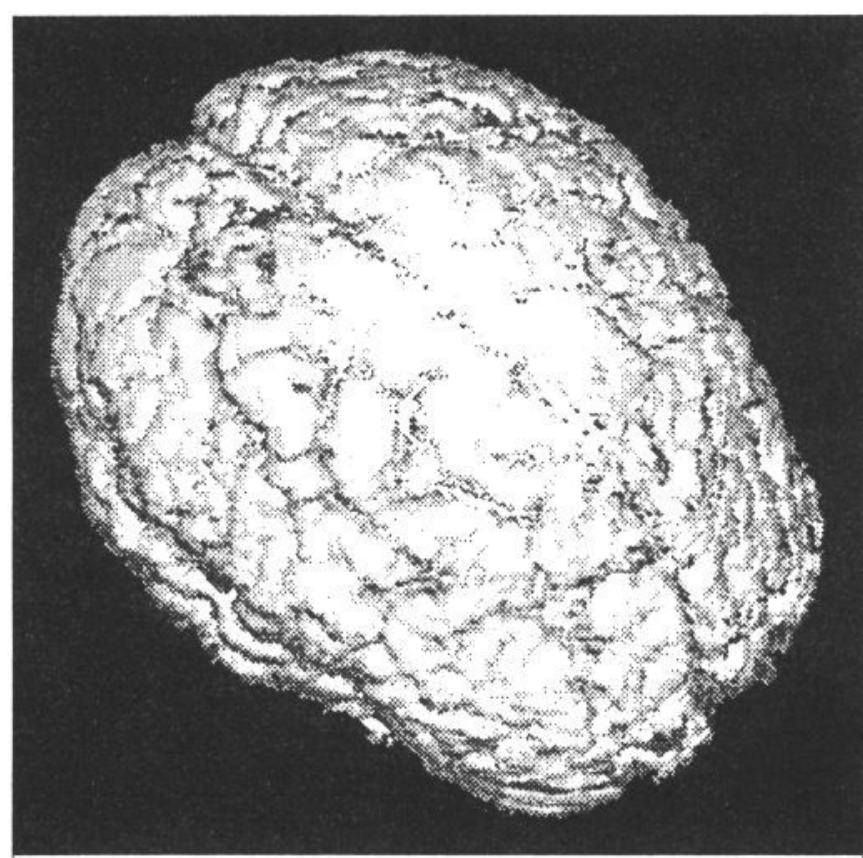

Figure 9. Voxel model of segmented cortical surface. View: Dorsal - Anterior. "fan" of pseudo slices, (rather than parallel coronal or sagittal slices) orthogonality between the slice plane and sulcus orientation, can be maintained.

The cortex edge located along each radial in the axial data was used to instantiate a vertical slice cortex description, and the DoG of each vertical slice obtained. The sulcus location algorithm was then applied (without modification) to locate sulci in this new orthogonal data set. The sulci locations found in the two orthogonal views can be OR-ed and displayed in the form of a side view map of the brain (see Figure 8). This map provides a basis for labelling the surface folds of sulci.

\section{Visualising the Cortical Surface and its Sulci}

The cortex descriptions obtained from the axial slice data have been used to segment the brain from its surrounding tissue and bone. This information has been used to create a surface shaded voxel model of the brain's surface (see Figure 9) revealing evidence of sulci and gyri on its surface. Sulci axis descriptions were also used to reconstruct a $3 \mathrm{D}$ voxel model of their structure in isolation from their surrounding cortical tissue. Figure 10 shows a view of a voxel model of the sulci axis descriptions obtained from axial and vertical slice data, combined. 


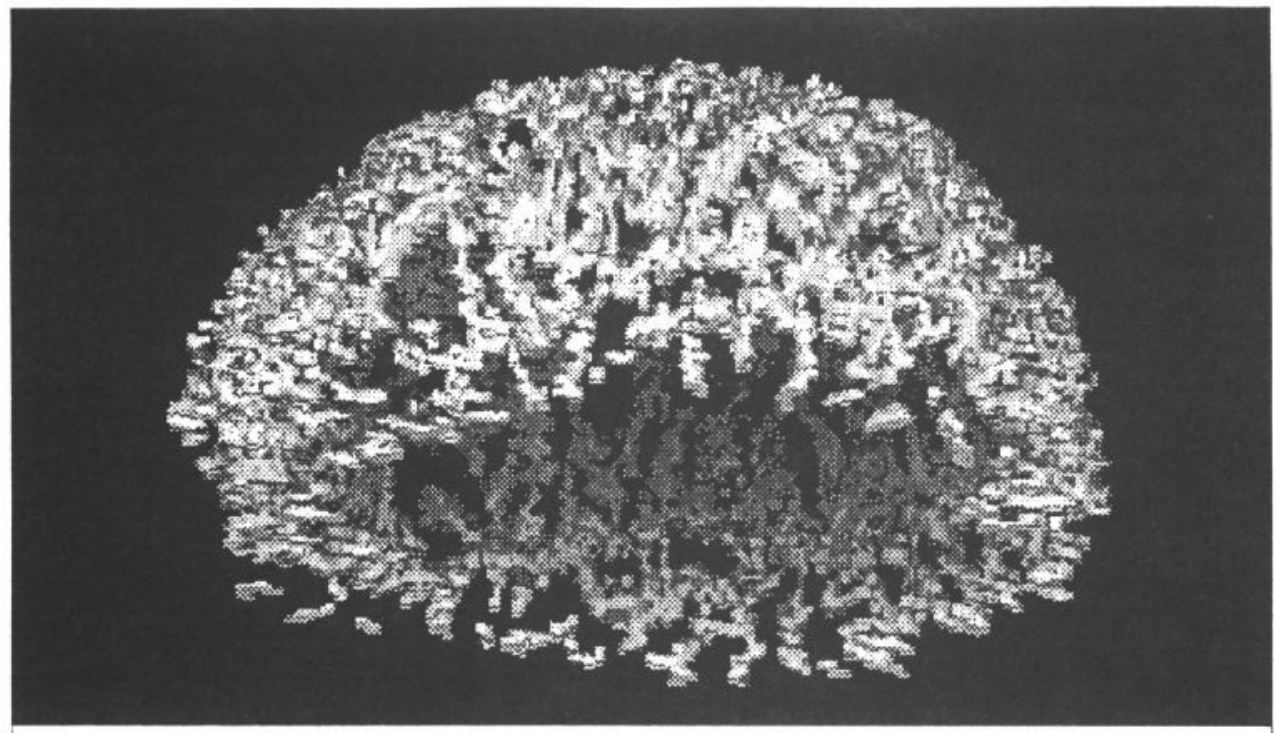

Figure 10. Left side view: the sulci internal axes extracted from the vertical and horizontal slice data.

\section{Discussion}

A review of cortex recognition methods in MR Images, reveals a number of common problems. In Attwood et al [1], zero crossings in Difference of Gaussian images plus heuristic knowledge about cortex geometry produced a closed line description of the outer cortex. These techniques suffered from missing data, confusion of inner and outer cortex surfaces and "leakage" into other structures. Region growing and texture segmentation techniques have also been applied to cortex recognition in MR images. The essential problem is that there are many cases where there is no local difference in intensity or texture between brain/cortex and other tissues, so that any knowledge-free, locally-based technique is likely to fail.

Intensity based segmentation, has problems as there is often little difference in intensity between the brain and other tissues, so that "leakage" problems occur. In systems which use more sophisticated tissue modelling techniques [6], skilled operator intervention is required, to teach the system appropriate grey values/ranges for different tissue types. Gerig et al [5] had some success using an automatic brain segmentation based on multi-spectral statistical classification in dual-echo MR sequences, but the system failed on images in which strict acquisition quality standards were not met, and no object recognition was attempted. Brummer et al [3] segmented brain contours automatically using histogram-based thresholding, followed by morphological operations to remove small regions and fine detail, then a distance transformation to pick out cerebral structures and finally propagation of results between slices. Their results were good for a large number of images tested but suffered some leakage and partial volume problems.

Our automatic method was designed to operate upon standard, noisy, clinical quality MR images which have not been selected on the basis of good contrast between the tissues of interest. This work represents the first attempt, that we are aware of, within the literature to explicitly detect internal sulci structures. The cortex recognition algorithm does suffer occasional leakage problems (especially into fatty tissue), but these events are rare and localised and do not appear to effect the sulci location algorithm's performance. 


\section{Conclusion}

We have described a new approach to locating the main cortical structures in MR images, using a relaxation process based on a specially tailored statistical edge detector. The main sulci and gyri have been detected. Current work is directed towards linking sulci together across slice levels and labelling these structures in terms of an anatomical model. Preliminary work suggests that computing the degree of overlap between 2D descriptions of sulci axes recovered in adjacent slices can provide powerful evidence as to which sulci should link with which. Further replication of the main algorithms using new data sets is also in hand.

\section{Acknowledgments.}

This work was supported initially by Alvey project MMI-134 "Model-based Processing of Radiological Images". It is currently funded by the University of Reading Research Endowment Fund. We would also like to thank Guy's Hospital MR Unit for kindly supplying the MR data sets used.

\section{References}

1. Attwood C.I., Sullivan G. D., Robinson, G. P., Baker, K.D., \& Colchester, A.C.F. "Model-based Interpretation of Anatomical Structures in Cranial MR Images". Proc. BMVC. Oxford, England. 1990.

2. Berger M.S., Cohen W.A. \& Ojemann G.A. "Correlation of motor cortex brain mapping data with magnetic resonance imaging". Journal of Neurosurgery, 72, 383387.1990 .

3. Brummer M.E., Mersereau R.M., Eisner R L \& Lewine R.R.J. "Automatic Detection of Brain Contours in MRI Data Sets”. Proc. 12th Int.Conf. I.P.M.I, UK. In: Lecture Notes in Computer Science 511. Springer-Verlag. 1991.

4. Ebeling U., Steinmetz H., Huang Y. \& Kahn T. "Topography and identification of the Inferior Precentral Sulcus in MR Imaging”. American Journal of Roentgenology, 153 (5), 1051-1056. 1989.

5. Gerig G., Martin J., Kikinis R., Kübler O., Shenton M \& Ferenc A. J. "Automatic Segmentation of Dual-Echo MR Head Data". Proc. 12th Int.Conf, I.P.M.I, UK. In: Lecture Notes in Computer Science, Springer-Verlag. 1991.

6. Kline H. E., Lorenson W.E., Kikinis R. \& Jolesz, F. "Three-Dimensional Segmentation of MR Images of the Head Using Probability and Connectivity". Journal of Computer Assisted Tomography. 14(6), 1037-1045, 1990.

7. Sedat J. \& Duvernoy H. "Anatomical Study of the Temporal lobe: Correlations with nuclear magnetic resonance". Journal of Neuroradiology, 17, 26-49. 1990.

8. Steinmetz H., Ebeling U., Huang Y \& Kahn T. "Sulcus Topography of the Parietal Opercular Region: An anatomic and MR Study". Brain and Language, 38, $515-$ 533. 1990.

9. Steinmetz H., Furst G., \& Freund H-J. "Cerebral Cortical Localization: Application and Validation of the Proportional Grid system in MR Imaging". Journal of Computer Assisted Tomography. 13 (1) 10-19. 1989.

10. Steinmetz H., Rademacher J., Janke L., Huang Y., Thron A. \& Zilles K. "Total surface of Temoroparietal Intrasylvian Cortex: Diverging Left-Right Asymmetries". Brain and Language, 39, 357-372. 1990

11. Sullivan G.D., Worral A.D., Hockney, R.W. \& Baker K.D. "Active Contours in Medical Image Processing using a Networked SIMD Array Processor". Proc. BMVC, Oxford, England. 1990. 